\title{
Glycemic profile is improved by High Slowly Digestible Starch diet in type 2 diabetic patients
}

\author{
Aurelie Goux ${ }^{1}$, Anne-Esther Breyton ${ }^{1,2}$, Alexandra Meynier ${ }^{1}$, Stephanie Lambert-Porcheron ${ }^{2}$, \\ Monique Sothier ${ }^{2}$, Laurie Van Den Berghe ${ }^{2}$, Sylvie Normand ${ }^{2}$, Emmanuel Disse ${ }^{2,3}$, \\ Martine Laville ${ }^{2,3}$, Julie-Anne Nazare ${ }^{2}$ and Sophie Vinoy ${ }^{1}$ \\ ${ }^{1}$ Nutrition Research, Mondelez International, Saclay, France, \\ ${ }^{2}$ Centre de Recherche en Nutrition Humaine Rhône-Alpes, Univ-Lyon, CarMeN Laboratory, Université Claude Bernard \\ Lyon1, Hospices Civils de Lyon, F-CRIN/FORCE Network, Pierre Bénite, France and \\ ${ }^{3}$ Endocrinologie, Nutrition, Diabète, Hospices Civils de Lyon, Pierre Bénite, France
}

\begin{abstract}
Introduction

Considering the dramatically increasing incidence of type 2 diabetes (T2D), decreasing glycemic variability in T2D patients is a key challenge to limit the occurrence of diabetic complications. Diet appears as one potential lever that can be set up above medications. Particularly, the ingestion of foods with a high content in slowly digestible starch (SDS) demonstrated both lower postprandial glycemic and insulin responses in healthy and insulin resistant subjects. This study aimed at designing a full high-SDS diet by selecting high-SDS starchy food products and at studying its impact on glycemic response and variability in T2D.
\end{abstract}

Materials and methods

This pilot randomized controlled cross-over study included eight T2D patients $\left(\mathrm{HbA} 1 \mathrm{c}=7.0 \pm 0.2 \%, \mathrm{BMI}=31.7 \pm 2.1 \mathrm{~kg} / \mathrm{m}^{2}, \mathrm{treated}\right.$ by Metformin \& Sitagliptin) who consumed twice, for one week a controlled diet containing starchy food products screened and selected to be either High (High-SDS) or Low (Low-SDS) in SDS, as determined by the SDS in-vitro method developed by Englyst et al. During each diet period, the glycemic profile was monitored for 6 days using a Continuous Glucose Monitoring System (CGMS). Multiple metrics related to variability and glycemic responses were calculated.

\section{Results}

222 SDS analyses were realized on commercial food products as consumed. 23 High-SDS and 20 Low-SDS food items with associated specific cooking instructions were selected to design two diets consistent with local T2D recommendations. The High-SDS diet demonstrated a significantly higher SDS content compared to the Low-SDS diet (61.6 vs $11.6 \mathrm{~g} /$ day; $\mathrm{p}<0.0001)$, mainly driven by selected pasta, rice and high-SDS biscuits (75.6\% of the consumed SDS content). The $\%$ of total daily energy intake (TDEI) for all macronutrients remained similar between diets $(\mathrm{p}>0.05)$ and the carbohydrate content specifically represented $49 \pm 1 \%$ and $47 \pm 2 \%$ of the TDEI for High-SDS and Low-SDS diets, respectively. With the high-SDS diet, the Mean Amplitude of Glycemic Excursion, a key parameter of glycemic variability, was significantly decreased (79.6 for Low-SDS vs $61.6 \mathrm{mg} / \mathrm{dL}$ for High-SDS; $\mathrm{p}$ $=0.0067)$. The significant correlation between the meals SDS contents and various glycemic parameters such as postprandial iAUC, tAUC (up to $180 \mathrm{~min}$ ) or peak value strengthen this finding $(\mathrm{p}<0.05$ for all).

\section{Discussion}

It was the first demonstration that a diet including selected starchy food items and cooking recommendations designed to favor products' high SDS content beneficially impacts glycemic profile in T2D subjects. Carefully selecting starchy food may be a simple and valuable tool to improve glycemic control in T2D.

\section{Conflict of Interest}

Aurélie Goux, Alexandra Meynier and Sophie Vinoy are employed by Mondelez International. Anne-Esther Breyton PhD is partially financed by Mondelez International. 